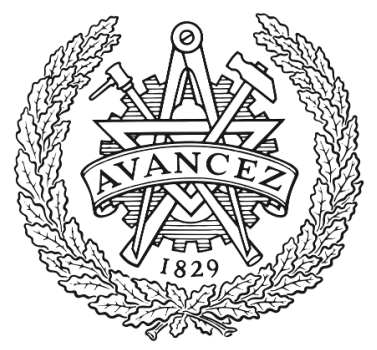

CHALMERS

UNIVERSITY OF TECHNOLOGY

\title{
Novel Millimeter Wave Transition From Microstrip Line to Groove Gap Waveguide for MMIC Packaging and Antenna Integration
}

Downloaded from: https://research.chalmers.se, 2023-04-26 14:25 UTC

Citation for the original published paper (version of record):

Nandi, U., Uz Zaman, A., Vosoogh, A. et al (2017). Novel Millimeter Wave Transition From Microstrip Line to Groove Gap Waveguide for MMIC

Packaging and Antenna Integration. IEEE Microwave and Wireless Components Letters, 27(8): 691-693. http://dx.doi.org/10.1109/LMWC.2017.2723679

N.B. When citing this work, cite the original published paper.

C2017 IEEE. Personal use of this material is permitted.

However, permission to reprint/republish this material for advertising or promotional purposes 


\title{
Novel Millimeter Wave Transition from Microstrip line to Groove Gap Waveguide for MMIC packaging and Antenna Integration
}

\author{
Uttam Nandi, Ashraf Uz Zaman, Member, IEEE, Abbas Vosoogh, and Jian Yang, Senior Member, IEEE
}

\begin{abstract}
In this paper a new microstrip line to groove gap waveguide transition has been proposed. The transition is based on a backshort cavity, which efficiently helps to couple the field from microstrip line to groove gap waveguide. In this transition the microstrip section lies inside the groove gap waveguide. Such contactless transition inside the waveguide is advantageous as it will allow easy packaging and integration of millimeter wave circuitry with gap waveguide components such as filters and array antennas. Alumina $\left(\epsilon_{r}=9.9\right)$ was used as the microstrip substrate for the proposed transition in this work. Measured results for the V-band transition show a relative bandwidth of $26 \%$ for a return loss better than $10 \mathrm{~dB}$. The maximum insertion loss of the manufactured back-to-back prototype is found to be 1.32 dB, which also includes the losses of a $5.45 \mathrm{~mm}$ long microstrip line on an Alumina substrate. After subtracting the losses in the microstrip section, the losses in a single transition is found to be varying between 0.145 and $0.38 \mathrm{~dB}$.
\end{abstract}

Keywords-Groove Gap Waveguide (GGW), Microstrip line, Millimeter Wave (mmW), Printed Circuit Board (PCB).

\section{INTRODUCTION}

There is a growing trend in utilizing millimeter wave $(\mathrm{mmW})$ frequencies in various fields such as telecommunication, automotive and transportation, health care and defense. Such $m m W$ technologies should be compact, portable and self-configurable for easy installation in various places. However, there are several issues with $m m W$ components and circuits. Usually, expensive waveguide assembly and fabrication techniques increase the cost of waveguide components. Also, it becomes more and more challenging to integrate RF electronics with the waveguide components by using the traditional wirebond or flip-chip based interconnect technologies. This limits the extensive use of $m m W$ frequencies. Gap wave technology provides attractive solutions to overcome some of these limitations of millimeter waves [1]. Use of the gap wave technology for making high-Q bandpass filters and packaging of MMIC circuits have already been done [2], [3]. Gap waveguide has been used for producing high gain array antennas, which overcomes the high frequency limitations of traditional waveguides such as microstrip and rectangular waveguide [4]-[6]. As mentioned in the above mentioned works, the split block architecture of the gap waveguide is ideal for packaging of $m m W$ circuitry. Integrating $\mathrm{mmW}$ circuitry into the gap waveguide components and array antennas will make the entire $\mathrm{Tx} / \mathrm{Rx}$ module more compact. Beam steering and massive MIMO can be implemented by integrating MMIC based active circuits into the feed-network of gap waveguides array antennas. Hence, designing a suitable transition from microstrip to gap waveguide is of immense importance. Several transitions have been designed so far for different gap waveguides. Transition from ridge gap waveguide to microstrip had been done earlier using pressure contact in [7]. However, it has the constrain that the thickness of the substrate has to be same as that of the air gap of the ridge gap waveguide. Moreover, a pressure based galvanic contact becomes extremely difficult for $\mathrm{mmW}$ frequencies and it could also damage the circuitry. Transition through EM coupling by overlapping quarter wavelength of ridge with patch have also been investigated in [8], [9]. However, this transition was very sensitive and showed very narrow band performance. In this letter a new and robust groove gap waveguide (GGW)-microstrip transition using a resonant cavity has been proposed. The proposed approach is very different compared to the traditional rectangular waveguidemicrostrip backshort transitions where the microstrip and the waveguide are in perpendicular planes [10], [11]. Hence, traditional backshort transitions occupies more space and have several packaging issues such as leakage. In this proposed transition the microstrip is inside the GGW which provides a simple and compact solution for packaging $m m W$ circuitry.

\section{Proposed Transition and Simulation Results}

The schematic of the proposed GGW-microstrip transition is shown in Fig. 1 and the details of the microstrip section is shown in Fig. 2. The operating principle of the transition is based on the EM coupling with the help of a cavity and the proposed transition is composed of three sections: a metal cavity, input coupling section for the cavity and the output coupling section for the cavity. The cavity is a half wavelength resonant cavity and the depth of this cavity is about $\lambda / 4$. A microstrip line with a tapered probe section is placed above the cavity which couples the $Q-T E M$ mode of the microstrip line to the cavity. It is important to note that, the part of the substrate which is extended over the cavity does not have a ground plane. This is considered as an input coupling section for the cavity. Similarly, a narrow section of the groove gap waveguide also couples the $Q-T E_{10}$ mode of the groove gap waveguide to the cavity and this section could be considered as the output coupling section for the same cavity. As seen in Fig. 1 there is a block just after the rectangular cavity which acts as a capacitive iris. Also, the first set of pins just after the cavity is extended into the GGW which is used to make the narrow section of the groove gap waveguide. Impedance matching between the GGW and microstrip line is achieved 
a)

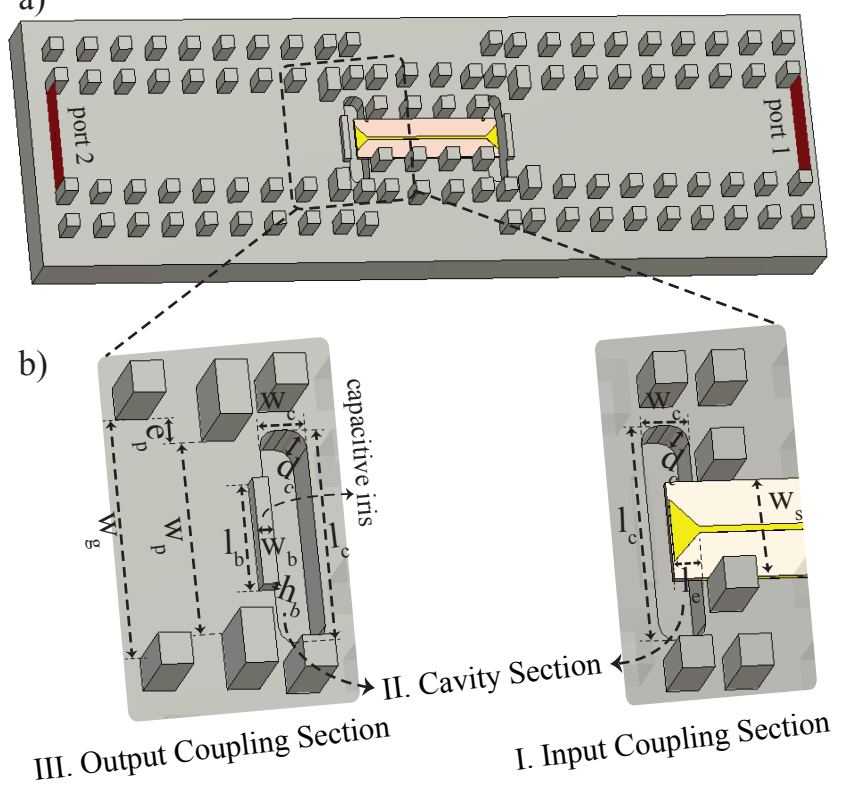

Fig. 1. a) Back-to-back GGW-microstrip transition (top metal plate hidden). b) 3 sections for the proposed GGW-microstrip transition and its optimised dimensions $\left(w_{g}=3.8 \mathrm{~mm}, w_{c}=0.73 \mathrm{~mm}, l_{c}=3.39 \mathrm{~mm}, d_{c}=\right.$ $0.91 \mathrm{~mm}, w_{b}=0.24 \mathrm{~mm}, l_{b}=1.69 \mathrm{~mm}, h_{b}=0.31 \mathrm{~mm}, e_{p}=$ $\left.0.37 \mathrm{~mm}, w_{p}=3.06 \mathrm{~mm}, l_{e}=0.45 \mathrm{~mm}, w_{s}=1.49 \mathrm{~mm}\right)$.

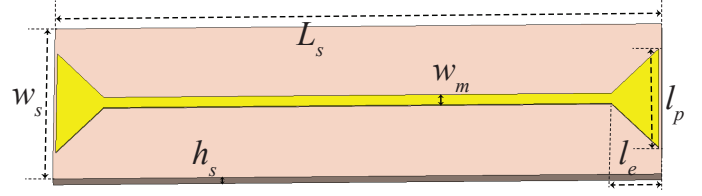

Fig. 2. PCB design for GGW to microstrip transition and its dimensions after optimization $\left(L_{s}=5.45 \mathrm{~mm}, w_{s}=1.49 \mathrm{~mm}, h_{s}=0.127 \mathrm{~mm}\right.$, $l_{e}=0.45 \mathrm{~mm}, w_{m}=0.1 \mathrm{~mm}, l_{p}=0.98 \mathrm{~mm}$ )

by tuning the dimensions of the cavity, the dimensions of the tapered probe, the dimensions of the capacitive irises and the width of the narrow groove gap waveguide section. The width of the microstrip line $\left(w_{m}=0.1 \mathrm{~mm}\right)$ is chosen such that the impedance of the line is $50 \Omega$. The depth of the cavity is $\lambda / 4 \sim\left(d_{c}=0.91 \mathrm{~mm}\right)$. For impedance matching the length of cavity is kept at $l_{c}=3.39 \mathrm{~mm}$ which is comparable with the width of the groove $\left(w_{g}=3.8 \mathrm{~mm}\right)$. After optimization, the width of the narrow section of groove gap waveguide is kept $w_{p}=3.06 \mathrm{~mm}$. It is also worth mentioning that there is a limit to the maximum width of the PCB for which a good transition can be made. If the substrate is made bigger, there will be leakage due to the existence of the higher order mode in the substrate. This is because the waves can propagate as $T E_{10}$ modes if the width is more than $\lambda / 2$. Hence if transition with larger substrate width is required, additional pins from the top metal plate above the substrate will be needed to suppress the unwanted modes in the substrate [12], [13]. In this proposed design the width of the PCB is $\left(w_{s}=1.49 \mathrm{~mm}\right)$ less than

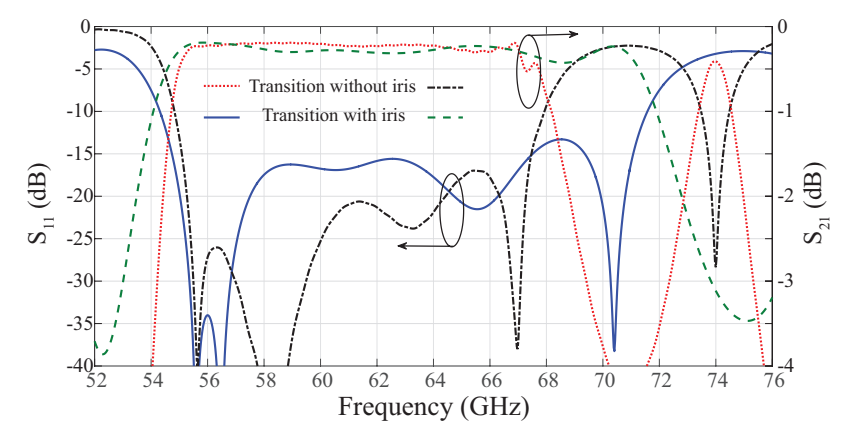

Fig. 3. S-parameters for two different back-to-back GGW-Microstrip transitions, one optimised without using block and the final transition using a block just after the cavity.

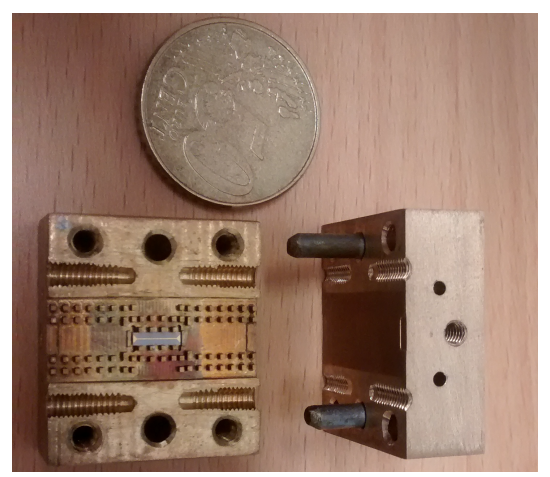

Fig. 4. Manufactured GGW Prototype with the PCB integrated inside the prototype.

$\lambda / 2$ and hence there is no leakage problem.

Millimeter wave circuitry such as RF MMICs have typically high substrate permittivity. Hence, Alumina $\left(\epsilon_{r}=9.9\right)$ substrate is considered for the microstrip line in the proposed transition. Initially, GGW-microstrip transition was optimised without a capacitive iris just after the cavity. However, the final transition was done by using a capacitive iris. Fig. 3 shows the S-parameters for the back-to-back GGW-microstrip transitions with and without the capacitive iris. One can see that the transition without the iris has better matching in a narrower band whereas, the transition with the iris has wider bandwidth for $S_{11}$ below -15 dB. Therefore, the transition with the iris is selected as the final one to cover the frequency range of $54.2-71.6 \mathrm{GHz}$ for a return loss better than $10 \mathrm{~dB}$. This is approximately $27.75 \%$ relative bandwidth. The simulated insertion loss is better than $0.5 \mathrm{~dB}$ for the frequency band.

\section{Measured Results}

The final prototype of the GGW-microstrip transition was manufactured using a computer numerical control (CNC) milling machine. The prototype was made in brass so that the PCB can be soldered to brass surface easily. The PCB was manufactured by standard photo-lithography process and the substrate used was alumina. Fig. 4 shows the manufactured prototype for the GGW-microstrip transition with the PCB 


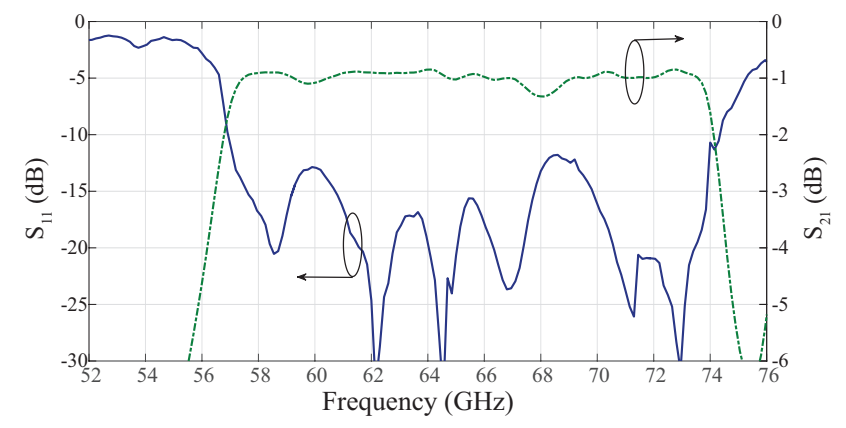

Fig. 5. Measured S-parameters for the proposed back-to-back GGWmicrostrip transition.

integrated inside the GGW. The prototype has been measured with Agilent PNA (N522A) and VDI extender modules for Vband. The measured S-parameters is presented in Fig. 5. The measured GGW-microstrip transition works for a frequency range of $56.7-74.1 \mathrm{GHz}$. As it can be seen from the plot, the measured results for the back-to-back prototype has been shifted towards the high frequency band and the $S_{11}$ level is higher than the simulation. The reason for this was investigated, and on checking the dimensions of the manufactured prototype it was observed that the manufactured cavity in the prototype was slightly smaller than the simulated dimensions. Apart from this, there are possible errors in alignment and placement of the PCB within the metal block and this can also degrade the matching and hence may cause higher level of $S_{11}$. The measured insertion loss as seen in Fig. 5 vary between $0.85-1.32 \mathrm{~dB}$. This includes the losses in the metal waveguide blocks and also the losses in the $5.45 \mathrm{~mm}$ microstrip line. The losses in the microstrip line with alumina substrate has been experimentally found out to be $0.103 \mathrm{~dB} / \mathrm{mm}$ in [13]. After taking away the losses in the microstrip line, the loss for single transition varies between $0.145-0.38$ $\mathrm{dB}$. The measured insertion loss is more than the simulated insertion loss, which is attributed to the degradation of metal conductivity due to surface roughness which was not taken into account in simulation. Also, the $\tan \delta$ of the alumina substrate used in simulation was taken from datasheet where it was specified at $10 \mathrm{GHz}$. In reality, the $\tan \delta$ is higher at $\mathrm{mmW}$ frequencies. However, the overall measured S-parameters were in acceptable limits and in reasonable agreement with the simulated results.

\section{CONCLUSION}

A novel microstrip to groove gap waveguide transition has been proposed in this work. This transition utilises a resonant cavity of depth $\lambda / 4$, which helps to couple the fields from the microstrip line to the groove gap waveguide. This transition is compact and the microstrip lies inside the GGW structure. Hence, this is a good solution for packaging of millimeter wave circuitry. The proposed GGW-microstrip transition had been manufactured and the measured results are in reasonable agreement with the simulated results. This novel transition scheme is very suitable for a multifunctional MMIC integration and the integration can be performed without the need of RF bonding or flipchip interconnection, which is a big advantage compared to todays state of the art technique.

\section{ACKNOWLEDGMENT}

This work is supported by European Research Council (ERC) via an advanced investigator grant ERC - $2012-$ $A D G \_20120216$ and Swedish Governmental Agency for Innovation Systems (VINNOVA) grant- 2015-01387 within the Smart Electronics Program.

\section{REFERENCES}

[1] P.-S. Kildal, E. Alfonso, A. Valero-Nogueira, and E. Rajo-Iglesias, "Local metamaterial-based waveguides in gaps between parallel metal plates," IEEE Antennas and Wireless Propagation Letters, vol. 8, pp. 84-87, 2009.

[2] E. A. Alós, A. U. Zaman, and P.-S. Kildal, "Ka-band gap waveguide coupled-resonator filter for radio link diplexer application," IEEE Transactions on Components, Packaging and Manufacturing Technology, vol. 3, no. 5, pp. 870-879, 2013.

[3] A. U. Zaman, Gap waveguide: Low loss microwave passive components and MMIC packaging technique for high frequency applications. Chalmers University of Technology, 2013.

[4] A. Vosoogh and P.-S. Kildal, "Corporate-fed planar $60 \mathrm{GHz}$ slot array made of three unconnected metal layers using AMC pin surface for the gap waveguide," IEEE Antennas and Wireless Propagation Letters, 2015 .

[5] H. Kirino and K. Ogawa, "A $76 \mathrm{GHz}$ multi-layered phased array antenna using a non-metal contact metamaterial waveguide," IEEE Transactions on Antennas and Propagation, vol. 60, no. 2, pp. 840-853, 2012.

[6] D. Zarifi, A. Farahbakhsh, A. Zaman, and P.-S. Kildal, "Design and Fabrication of a High-Gain $60 \mathrm{GHz}$ Corrugated Slot Antenna Array with Ridge Gap Waveguide Distribution Layer," IEEE Transactions on Antennas and Propagation, vol. 64, pp. 2905-2913, 2016.

[7] A. U. Zaman, T. Vukusic, M. Alexanderson, and P.-S. Kildal, "Design of a simple transition from microstrip to ridge gap waveguide suited for MMIC and antenna integration," IEEE Antennas and Wireless Propagation Letters, vol. 12, pp. 1558-1561, 2013.

[8] A. Algaba Brazález, A. U. Zaman, and P.-S. Kildal, "Investigation of a Microstrip-to-Ridge Gap Waveguide transition by electromagnetic coupling," in IEEE Antennas and Propagation Society, AP-S International Symposium (Digest), 2012, pp. 1-2.

[9] A. A. Brazález, J. Flygare, J. Yang, V. Vassilev, M. Baquero-Escudero, and P.-S. Kildal, "Design of-Band Transition From Microstrip to Ridge Gap Waveguide Including Monte Carlo Assembly Tolerance Analysis," IEEE Transactions on Microwave Theory and Techniques, vol. 64, no. 4, pp. 1245-1254, 2016.

[10] Y.-C. Leong and S. Weinreb, "Full band waveguide-to-microstrip probe transitions," in Microwave Symposium Digest, 1999 IEEE MTT-S International, vol. 4. IEEE, 1999, pp. 1435-1438.

[11] K. Sakakibara, M. Hirono, N. Kikuma, and H. Hirayama, "Broadband and planar microstrip-to-waveguide transitions in millimeter-wave band," in Microwave and Millimeter Wave Technology, 2008. ICMMT 2008. International Conference on, vol. 3. IEEE, 2008, pp. 1278-1281.

[12] J. Zhang, X. Zhang, D. Shen, and K. Wu, "Gap Waveguide PMC Packaging for a SIW-GCPW-Based Filter," IEEE Microwave and Wireless Components Letters, vol. 26, no. 3, pp. 159-161, 2016.

[13] A. U. Zaman, V. Vassilev, P.-S. Kildal, and H. Zirath, "Millimeter Wave E-Plane Transition From Waveguide to Microstrip Line With Large Substrate Size Related to MMIC Integration," IEEE Microwave and Wireless Components Letters, vol. 26, no. 7, pp. 481-483, 2016. 